\title{
Intensity Non-uniformity Correction of Magnetic Resonance Images Using a Fuzzy Segmentation Algorithm
}

\author{
S. Shen ${ }^{1}$, W. A. Sandham ${ }^{2}$, M. H. Granat ${ }^{3}$, A. Sterr \\ ${ }^{1}$ Department of Psychology, University of Surrey, UK \\ ${ }^{2}$ Scotsig, Glasgow, UK \\ ${ }^{3}$ Glasgow Caledonian University, Glasgow, UK
}

\begin{abstract}
Artifacts in magnetic resonance images can make conventional intensity-based segmentation methods very difficult, especially for the spatial intensity non-uniformity induced by the radio frequency (RF) coil. The non-uniformity introduces a slow-varying shading artifact across the images. Many advanced techniques, such as nonparametric, multi-channel methods, cannot solve the problem. In this paper, the extension of an improved fuzzy segmentation method, based on the traditional fuzzy c-means (FCM) algorithm and neighborhood attraction, is proposed to correct the intensity non-uniformity. Experimental results on both synthetic non-MR and MR images are given to demonstrate the superiority of the algorithm.
\end{abstract}

\section{INTRODUCTION}

Magnetic resonance imaging (MRI) is an advanced medical imaging technique, which provides rich information about human anatomical structure due to its high spatial resolution and excellent discrimination of soft tissues. Brain is the most complex organ in the human body, and therefore segmentation of brain MR images is an important technique for facilitating an image-based diagnosis, especially for the analysis of brain tumors. Unfortunately, manual segmentation of different tissues is very time-consuming due to the large amount of data involved. A number of supervised and unsupervised techniques have therefore been proposed in recent years for automatic segmentation of MR images. However, their effectiveness is deteriorated by spatial intensity non-uniformity, also called the bias field, induced by the radio frequency $(\mathrm{RF})$ used during MRI acquisition $[1,2]$. This is a major problem for automated segmentation techniques. Specifically, these artifacts have made conventional intensity-based segmentation very difficult, since the statistics of tissue intensities are spatially altered by the bias field. A wide variety of approaches have therefore been developed for intensity non-uniformity correction. Tincher et al [3] modeled the bias field using a second-order polynomial and fitted it to a uniform phantom-scanned MRI. However, it required the geometry relationship between the coils and the image data, which is not usually available. Wells et al [4] presented a method called adaptive segmentation to correct and segment MR images, using the expectation-maximization (EM) algorithm. This is a very popular method for non-uniformity correction and is used by many other researchers [5]. However, there are two main disadvantages of EM approaches. First, the EM algorithm is computationally intensive, especially for large data sets. Second, the EM algorithm requires a reasonable initial guess for either the bias field or the clustering estimate; otherwise the algorithm could become easily trapped in a local minimum. In this paper, a different approach using a fuzzy clustering technique is described. It is based on the traditional fuzzy c-means (FCM) clustering algorithm and considers neighborhood attraction to correct the intensity non-uniformity during segmentation.

The paper is organized as follows. Section II describes the methodology of the procedure. Section III then validates the method using synthetic non-MR and MR images. Conclusions are summarized in Section IV.

\section{METHODOLOGY}

\section{A. Background}

Let $X=\left\{X_{1}, X_{2}, \cdots, X_{j}, \cdots, X_{N}\right\}$ be the observed MRI intensities (with artifacts) and $X^{*}=\left\{X_{1}^{*}, X_{2}^{*}, \cdots, X_{j}^{*}, \cdots, X_{N}^{*}\right\}$ be the ideal intensities (without artifacts). $N$ is the number of pixels in the image. The bias field is denoted by 
$B=\left\{B_{1}, B_{2}, \cdots, B_{j}, \cdots, B_{N}\right\}$. The observed MRI intensity is modeled as a product of the true signal generated by the underlying anatomy and the bias field, expressed as follows:

$$
X_{j}=X_{j}^{*} \times B_{j} \quad(j=1 \ldots N)
$$

The application of a logarithmic transformation to the intensities allows the bias field to be modeled as an addition to the ideal intensities. Let $\mathbf{x}_{j}$ and $\mathbf{x}_{j}^{*}$ denote the observed and ideal log-transformed intensities of the $j$-th pixel. $\beta_{j}$ is the log-transformed bias field of the $j$-th pixel. So,

$$
\mathbf{x}_{j}=\mathbf{x}_{j}^{*}+\beta_{j}
$$

$\beta_{j}$ is modeled by an $N$-dimensional zero-mean Gaussian prior probability density, which is one of the most successful models $[2,4,5]$. It is defined as follows,

$$
\frac{1}{N} \sum_{j=1}^{N} \beta_{j}=0
$$

\section{B. Improved Fuzzy Segmentation Method}

The improved fuzzy c-means clustering (IFCM) algorithm is a segmentation method which was proposed to overcome noise effects in MR images. Instead of modifying the objective function in the traditional FCM algorithm [6], it improves the similarity measurement of the pixel intensity and the cluster centre by considering neighborhood attraction. Details of the algorithm can be found in $[7,8]$.

The objective function is expressed as follows:

$$
J_{m}(U, V)=\sum_{j=1}^{N} \sum_{i=1}^{C} \mathbf{u}_{i j}^{m} d^{2}\left(\mathbf{x}_{j}, \mathbf{v}_{i}\right)
$$

where $\mathbf{x}_{j}$ represents the pixel intensity of the $j$-th voxel and $\mathbf{u}_{i j} \subseteq U$ is its membership function to the $i$-th cluster, which satisfies $\mathbf{u}_{i j} \in[0,1], \sum^{C} \mathbf{u}_{i j}=1$.

$V=\left\{\mathbf{v}_{1}, \mathbf{v}_{2}, \cdots, \mathbf{v}_{i}, \cdots, \mathbf{v}_{C}^{i \neq 1}\right\}$ denotes the intensity center of the clusters, and $C$ is the total number of clusters in the image.

$d^{2}\left(\mathbf{x}_{j}, \mathbf{v}_{i}\right)$ was described as a similarity measurement between the pixel intensity and the cluster center in FCM. Since the intensity of a pixel should be identical to its neighboring pixels, an attraction is supposed to exist between those pixels nearby. Therefore, $d^{2}\left(\mathbf{x}_{j}, \mathbf{v}_{i}\right)$ was modified in IFCM as follows,

$$
d^{2}\left(\mathbf{x}_{j}, \mathbf{v}_{i}\right)=\left\|\mathbf{x}_{j}-\mathbf{v}_{i}\right\|^{2}\left(1-\lambda H_{i j}-\xi F_{i j}\right)
$$

where $H_{i j}$ and $F_{i j}$ are two factors of the neighbourhood attraction, called feature attraction and distance attraction. $\lambda$ and $\xi$ adjust the degree of attraction and have a magnitude between 0 and 1. $\|\cdot\|$ is regarded as a Euclidean distance.

\section{Bias Field Estimation}

Since the objective function must be minimized in IFCM, by considering the model in eq.(3), the constrained optimization may be expressed using Lagrange multipliers,

$$
J_{m}(U, V)=\sum_{j=1}^{N} \sum_{i=1}^{C} \mathbf{u}_{i j}^{m} d^{2}\left(\mathbf{x}_{j}, \mathbf{v}_{i}\right)+\eta\left(\frac{1}{N} \sum_{j=1}^{N} \beta_{j}\right)
$$

Taking the derivative of $J_{m}$ with respect to $\beta_{j}$ and setting the result to zero,

$$
\frac{\partial J_{m}}{\partial \beta_{j}}=\sum_{i=1}^{C} \mathbf{u}_{i j}^{m}(1-\lambda H-\xi F)^{2} \cdot 2\left\|\mathbf{x}_{j}-\beta_{j}-\mathbf{v}_{i}\right\|+\frac{\eta}{N}=0
$$

Let $M=1-\lambda H-\xi F$, and redefine $\eta=\frac{\eta}{N}$,

$$
\begin{gathered}
\sum_{i=1}^{C} \mathbf{u}_{i j}^{m} M^{2} \cdot 2\left\|\mathbf{x}_{j}-\beta_{j}-\mathbf{v}_{i}\right\|+\eta=0 \\
\beta_{j}=\mathbf{x}_{j}-\frac{\sum_{i=1}^{C} \mathbf{u}_{i j}^{m} M^{2} \mathbf{v}_{i}}{\sum_{i=1}^{C} \mathbf{u}_{i j}^{m} M^{2}}+\frac{\eta}{2 \sum_{i=1}^{C} \mathbf{u}_{i j}^{m} M^{2}}
\end{gathered}
$$

According to eq.(3),

$$
\sum_{j=1}^{N} \beta_{j}=\sum_{j=1}^{N} \mathbf{x}_{j}-\sum_{j=1}^{N} \frac{\sum_{i=1}^{C} \mathbf{u}_{i j}^{m} M^{2} \mathbf{v}_{i}}{\sum_{i=1}^{C} \mathbf{u}_{i j}^{m} M^{2}}+\sum_{j=1}^{N} \frac{\eta}{2 \sum_{i=1}^{C} \mathbf{u}_{i j}^{m} M^{2}}=0
$$

$$
\eta=\frac{\sum_{j=1}^{N}\left(\frac{\sum_{i=1}^{C} \mathbf{u}_{i j}^{m} M^{2} \mathbf{v}_{i}}{\sum_{i=1}^{C} \mathbf{u}_{i j}^{m} M^{2}}-\mathbf{x}_{j}\right)}{\sum_{j=1}^{N}\left(2 \cdot \sum_{i=1}^{C} \mathbf{u}_{i j}^{m} M^{2}\right)^{-1}}
$$

Substituting eq.(10) into eq.(8), the bias field may be estimated as follows,

$$
\beta_{j}=\mathbf{x}_{j}-p_{j}-\frac{\sum_{j=1}^{N}\left(\mathbf{x}_{j}-p_{j}\right)}{\sum_{i=1}^{C} \mathbf{u}_{i j}^{m} M^{2} \cdot \sum_{j=1}^{N}\left(\sum_{i=1}^{C} \mathbf{u}_{i j}^{m} M^{2}\right)^{-1}}
$$


Generally, the bias field is a very smooth field. It has always been modeled as a linear combination of smooth basis functions $[9,10]$. In this case, the bias field is smoothed by a two-dimensional Gaussian function,

$$
\beta_{j}=\beta_{j} * \exp \left(\frac{-\left(\mathbf{x}_{j}-\mu\right)^{2}}{\sigma^{2}}\right)
$$

The cluster intensity center of IFCM is modified as follows,

$$
\mathbf{v}_{i}=\frac{\sum_{j=1}^{N}\left(\mathbf{u}_{i j}\right)^{m}\left(\mathbf{x}_{j}-\beta_{j}\right)}{\sum_{j=1}^{N}\left(\mathbf{u}_{i j}\right)^{m}}
$$

\section{Implementation of the IFCM Algorithm}

The IFCM algorithm iteratively optimizes $J_{m}(U, V)$ with the continuous update of the membership function and the cluster center. The basic steps of this algorithm are given as follows:

1. Determine the number of clusters $C, 2 \leq C \leq N$.

2. Initialize the fuzzy membership $\mathbf{u}_{i j}^{(0)}$ of $\mathbf{x}_{j}$ belonging to the $i$-th cluster.

3. Initialize the bias field, $\beta_{j}=0$.

4. At the $l$-th iteration, calculate the cluster intensity centre $\mathbf{v}_{i}^{(l)}$ using eq.(13), $i=1,2, \cdots, C$.

5. Calculate the extended $d^{2}\left(\mathbf{x}_{j}, \mathbf{v}_{i}^{(l)}\right)$ in eq.(5).

6. Update $\mathbf{u}_{i j}^{(l)}$ with $d^{2}\left(\mathbf{x}_{j}, \mathbf{v}_{i}^{(l)}\right)$ as follows,

$$
\mathbf{u}_{i j}=\frac{1}{\sum_{k=1}^{c}\left(\frac{d\left(\mathbf{x}_{\mathbf{j}}, \mathbf{v}_{i}\right)}{d\left(\mathbf{x}_{j}, \mathbf{v}_{i}\right)}\right)^{\frac{2}{m-1}}}
$$

7. Estimate the bias field using eq.(11) and smooth it using a Gaussian function

8. Compare $\mathbf{u}_{i j}^{(l)}$ and $\mathbf{u}_{i j}^{(l-1)}$. If $\left\|\mathbf{u}_{i j}^{(l)}-\mathbf{u}_{i j}^{(l-1)}\right\|<\varepsilon$ then stop, otherwise $l=l+1$, go to Step 4 and repeat.

\section{RESULTS}

In this section, the improved fuzzy clustering method is applied to synthetic non-MR images corrupted with a multiplicative bias field, and simulated MR images with a $40 \%$ bias field. The parameters $\lambda$ and $\xi$ in eq.(5) were established as 0.47 and 0.53 , respectively, by trial and error.

Fig. 1(a) shows a two-class synthetic image corrupted by a Gaussian bias field. The segmentation results of FCM and IFCM are shown in Figs. 1(b) and 1(c), respectively. These demonstrate that the traditional FCM method was unable to

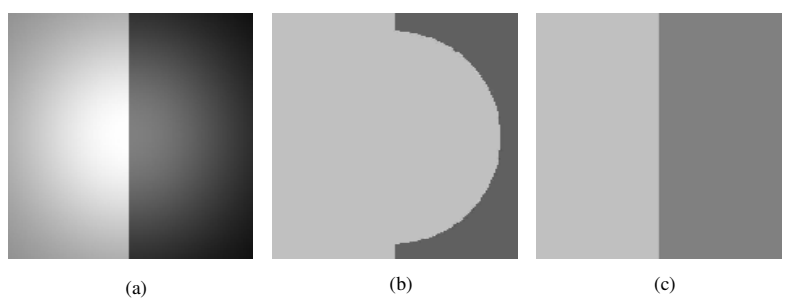

Figure 1 Synthetic image I. (a) Image corrupted by a Gaussian bias field. (b) FCM segmentation result. (c) IFCM segmentation result.

overcome the effect of the intensity non-uniformity, whereas IFCM segmentation achieved the correct result.

Another synthetic image with four classes is shown in Fig. 2(a). It also contained a Gaussian bias field. Fig. 2(b) displays the estimated bias field and 2(c) shows the corrected image.

To further demonstrate the ability of the extended IFCM method, a simulated MR image was downloaded from Brain Web (http://www.bic.mni.mcgill.ca/brainweb), a MRI simulated brain database [11-14]. It was a T1-weighted image with $1 \%$ noise and $40 \%$ non-uniformity. Fig. 3(a) shows the original simulated MR image and Fig. 3(b) shows the corrected image using the extended IFCM method. The image was segmented into four classes corresponding to background, gray matter, white matter and cerebral spinal fluid (CSF). The segmentation results of FCM and IFCM are given in Figs. 3(c) and 3(d), respectively. These demonstrate that the FCM algorithm was affected by the non-uniformity, while the extended IFCM algorithm not only succeeded in segmenting the image but also estimated the bias field (Fig. 3(e)).

However, using the extended IFCM algorithm specifically for the bias field estimation may lead to the loss of some image detail. Further work is required to solve this problem.

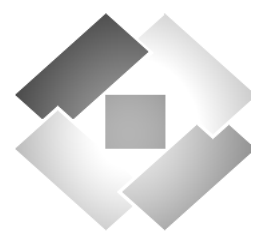

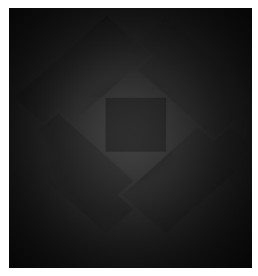

(b)

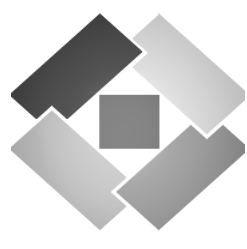

(c)
Figure 2 Synthetic image II for bias field estimation. (a) Image corrupted by a Gaussian bias field. (b) Estimated bias field. (c) IFCM segmentation result. 


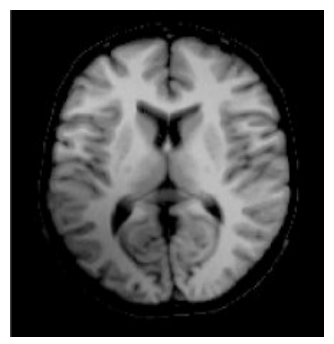

(a)

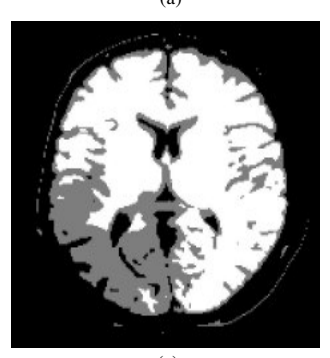

(c)

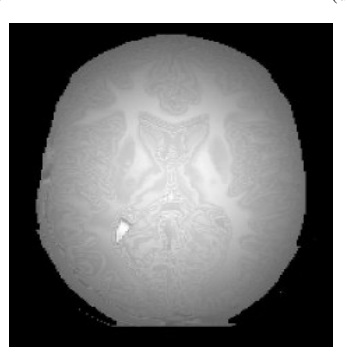

(e)

Figure 3 Simulated MR image. (a) Original image. (b) Corrected image. (c) FCM segmentation result. (d) IFCM segmentation result. (e) Estimated bias field

\section{CONCLUSIONS}

This paper has presented an improved fuzzy c-means clustering algorithm (IFCM) for the intensity non-uniformity correction of brain MR images. The traditional FCM algorithm is based on minimizing an objective function, defined by the difference between the intensity centers of the clusters and the signal intensities of pixels. However, MR images always include an intensity non-uniformity caused by the RF coil in MRI acquisition, which may introduce unexpected intensity values of the pixels. This may cause an inaccurate segmentation. The improved FCM algorithm was developed to solve this problem. It assumes that a neighborhood attraction exists between two neighboring pixels. During clustering, each pixel attempts to attract its neighboring pixels towards its own cluster. Therefore, in the IFCM algorithm, segmentation is not only decided by the pixel intensity, but also by its neighbors. The presence of the bias field changes the intensities of some pixels; these may be segmented more appropriately with the help of their neighbors. The bias field is modeled by a zero mean Gaussian field and estimated during the process of segmentation.

Different types of synthetic images with an intensity non-uniformity were employed for demonstration. The bias fields were corrected and estimated using IFCM. The segmentation results validated the superiority of IFCM over the traditional FCM algorithm. The drawback of IFCM for the bias field estimation is the loss of some image detail.

Future work will investigate reducing the loss of image detail during bias field estimation, and validating the IFCM algorithm on real MR images.

\section{REFERENCES}

[1] B. W. Dawant, A. P. Zijdenbos, and R. A. Margolin, "Correction of intensity variations in MR Images for computer-aided tissue classification," IEEE Trans. Medical Imaging, vol. 12, no. 4, pp. 770-781, 1993.

[2] M. N. Ahmed, S. M. Yamany, A. A. Farag and T. Moriarty, "A modified fuzzy c-means algorithm for bias field estimation and segmentation of MRI data," IEEE Trans. Medical Imaging, vol. 21, no. 3, pp. 193-199, 2002.

[3] M. Tincher, C. R.Meyer, R. Gupta and D. M. Williams, "Polynomial modeling and reduction of RF body coil spatial inhomogeneity in MRI," IEEE Trans. Medical Imaging, vol. 12, no. 2, pp. 361-365, 1993.

[4] W. M. Wells, III, W. E. L. Grimson, R. Kikinis and F. A. Jolesz, "Adaptive segmentation of MRI data," IEEE Trans. Medical Imaging, vol. 15 , no. 4, pp. 429-442, 1996.

[5] Y. Y. Zhang, M. Brady and S. Smith, "Segmentation of brain MR images through a hidden markov random field model and the expectation-maximization algorithm," IEEE Trans. Medical Imaging, vol. 20 , no.1, pp. 45-57, 2001.

[6] J. C. Bezek, Pattern Recognition with fuzzy object function algorithms, Plenum Press, 1981.

[7] S. Shen, W. A. Sandham, M. H. Grant, J. Patterson and M. F. Dempsey, "Fuzzy clustering based applications to medical image processing," Proc. IEEE EMBS 25th Annu. Int. Conf., pp. 747-750, 2003.

[8] S. Shen, W. A. Sandham, M. H. Grant, A. Sterr, "MRI fuzzy segmentation of brain tissue using neighborhood attraction with neural network optimization," IEEE Trans. Inf. Technol. Biomed., Accepted.

[9] J. G. Sled, B. Zijdenbos and A. C. Evans, "A nonparametric method for automatic correction of intensity nonuniformity in MRI data," IEEE Trans. Medical Imaging, vol. 17, pp. 89-97, 1998.

[10] K. V. Leemput, F. Maes, D. Vandermeulen, and P. Suetens, "Automated model based bias field correction of MR images of the brain," IEEE Trans. Medical Imaging, vol. 18, pp. 885-896, 1999.

[11] C. A. Cocosco, V. Kollokian, R. K. -S. Kwan and A.C. Evans, "BrainWeb: Online Interface to a 3D MRI Simulated Brain Database," NeuroImage, vol. 5, no. 4, part 2/4, S425, 1997.

[12] R. K. S. Kwan, A. C. Evans and G. B. Pike, "An Extensible MRI Simulator for Post-Processing Evaluation," Visualization in Biomedical Computing (VBC'96). Lect. Notes. Comput SC, vol. 1131, pp. 135-140, Springer-Verlag, 1996.

[13] R. K. S. Kwan, A. C. Evans and G. B. Pike, "MRI simulation-based evaluation of image-processing and classification methods," IEEE Trans. Med. Imag., vol. 18, no. 11, pp.1085-1097, 1999.

[14] D. L. Collins, A. P. Zijdenbos, V. Kollokian, J. G. Sled, N. J. Kabani, C. J. Holmes, and A. C. Evans, "Design and construction of a realistic digital brain phantom," IEEE Trans. Med. Imag., vol. 17, no. 3, pp.463-468, 1998. 